\title{
MARCO METODOLÓGICO PARA APLICAR UN MODELO DE GESTIÓN DE RIESGOS FINANCIEROS EN LA UNED
}

\author{
JORGE LUIS ARCE SOLANO \\ Universidad Estatal a Distancia, Costa Rica \\ jarces@uned.ac.cr
}

\section{RESUMEN}

Este artículo expone resultados de una investigación realizada en el 2013. Propone el Marco Técnico de Referencia para la Construcción de un Modelo de Gestión de Riesgos Financieros aplicado a una institución pública universitaria. Se analizan aspectos teóricos de la Gestión de Riesgos, muestra la propuesta del Estándar Australiano de Administración del Riesgo AS/NZS 4360:1999, que revela una serie de definiciones para comprender su aplicación en las organizaciones. La etapa metodológica consta de un objetivo central y tres objetivos específicos que exponen la ruta a seguir para construir el modelo de gestión. Se analizaron las características utilizadas para gestionar el riesgo en las empresas; dentro del análisis planteado, las acciones para determinar probabilidades (grado de ocurrencia del riesgo) e impactos (consecuencias que pueden generar el riesgo), el tratamiento que identifica técnicas y alternativas para la mitigación del riesgo y finalmente su seguimiento y monitoreo durante toda la gestión del riesgo. A partir de ahí, se propone el modelo a implementar.

PALABRAS CLAVE: NORMATIVA, RIESGOS, MODELO, ADMINISTRACIÓN INTEGRAL DE RIESGOS, FINANCIERO.
ABSTRACT

This paper offers a technical frame of reference in order to make a model for managing financial risk in a university. These are the results of a research carried out in 2013. Theoretical aspects of risk management are analyzed; it also shows the AS/NZS 4360:1999, Australian Standard of Risk Management, which brings a series of definitions in order to understand the way it is applied in organizations. Methodology has a central objective and three specific ones that show the route to follow in order to make the management model. Some elements were analyzed such asthe characteristics for business risk management and in doing so, the actions to determine probabilities were taken into account (risk level of occurrence) and impacts (consequences that may generate risk), techniques and alternatives to mitigate risk and finally how to follow them up during the whole process of risk management. From there on the model to implement proposed.

KEYWORDS: LAW, RISKS, MODEL, INTEGRAL RISK MANAGEMENT, FINANCIAL. 


\section{INTRODUCCIÓN}

La entrada en vigencia de la Ley 8292 de Control Interno (2002) y las Directrices Generales para el Establecimiento y Funcionamiento del Sistema Específico de Valoración de Riesgos Institucional (SEVRI, 2005) obligó a la Contraloría General de la República (CGR) y todos los órganos sujetos a fiscalización (incluido las universidades públicas) a disponer de un sistema de valoración de riesgo que identifique, analice y administre por medio de métodos o modelos previamente establecidos los riesgos que puedan atentar contra el buen funcionamiento institucional y el cumplimiento del ordenamiento jurídico nacional. Debido a esto, se investigó una alternativa para la implementación y aplicación de un marco técnico de referencia para la construcción de un modelo de gestión de riesgos en la Dirección Financiera de la Universidad Estatal a Distancia (UNED).

La investigación se desarrolló tomando en consideración el caso de la UNED, que al 2013 no evidenciaba la aplicación de lo indicado en la Ley 8292 de Control Interno. Los datos se obtuvieron por medio de la técnica directa de la entrevista, la observación documental y participante para describir, reconocer y analizar el conocimiento que tienen los colaboradores de la Dirección Financiera sobre el riesgo institucional y que se recolecta por medio de un taller realizado por el Consejo Nacional de Rectores (CONARE). Esa información será insumo para determinar la metodología mencionada para el modelo de gestión de riesgos de dicha dirección.

La propuesta busca establecer un canal de entrada para la aplicación de la ley mencionada. Además, el análisis y la reflexión sobre el control interno, por medio de acciones concretas de una gestión integral de riesgos que sensibilice y a la vez genere la necesidad de capacitarse en el tema en mención. Lo anterior considerando el conjunto de planes, normas, reglamentos, acciones, acuerdos, instrumentos y procedimientos que rigen el quehacer institucional.

Asimismo, la investigación busca responder la siguiente interrogante: ¿Cómo implementar una metodología para la construcción de un modelo de gestión de riesgos financieros en la universidad pública? Con lo anterior, se determinó que uno de los aspectos importantes a solventar dentro de la Dirección Financiera de la Universidad es el desarrollo y aplicación de una metodología para el modelo de gestión de riesgos financieros, que contribuya con la aplicación de la normativa vigente sobre control interno y que es regulada y supervisada por la CGR.

El objetivo de la investigación fue determinar el Marco Técnico de Referencia para la construcción de un Modelo de Gestión de Riesgos Financieros para la Dirección Financiera de la UNED. Se establecieron tres objetivos específicos; el primero determina los posibles riesgos en la Dirección Financiera; el segundo, fue analizar y evaluar los riesgos; por último, se propuso determinar los componentes estratégicos que debe contemplar el modelo. Para cumplir con cada uno de los objetivos, se desarrolló un marco conceptual que ayudó a establecer criterios técnicos necesarios para justificar el modelo, así como la metodología utilizada para la investigación, resultados y conclusiones finales para el cumplimiento de la propuesta.

La Dirección Financiera está integrada por cuatro oficinas: Presupuesto, Control de Presupuesto, Tesorería y Contabilidad. La construcción del modelo se generó mediante la recopilación de entrevistas y observando el comportamiento de exposiciones realizadas en un taller de Valoración de Riesgos realizado en CONARE. Este fue emitido a las cuatro universidades públicas del momento $y$, en cumplimiento con lo señalado en las políticas públicas establecidas por la ley 8292 de Control Interno y la Contraloría General de la República, quien es la encargada de fiscalizar 
el cumplimiento de esta norma en Costa Rica, buscando establecer responsables en el debido cumplimiento.

\section{MARCO TEÓRICO}

Para el fundamento de la propuesta, se estableció un marco teórico que recopila conceptos que justifiquen el modelo. En primera instancia, se considera el Estándar Australiano, el Comité de Basilea, Committee of Sponsoring Organizations (COSO) y, por último, de vital importancia, lo indicado en la ley General de Control Interno en Costa Rica y las diferentes entidades encargadas de brindarle seguimiento a la gestión de riesgos Está última, debido a que es la base de implementación para la mayoría de instituciones públicas similares a la UNED y que la Contraloría General de la República de Costa Rica (CGR), valora y solicita su implementación en cada entidad.

El Estándar Australiano es una guía para el establecimiento e implementación del proceso de administración de riesgos. Involucra el establecimiento del contexto y la identificación, el análisis, la evaluación, el tratamiento, la comunicación y el monitoreo en curso de los riesgos (Australino, 1999, pág. 3). Por su parte, COSO tiene como misión "mejorar la calidad de la información financiera mediante la ética en los negocios, los controles internos efectivos y el gobierno corporativo " (Ambrosone, La Administración del riesgo empresarial: Una responsabilidad de todos Enfoque COSO -, 2007, pág. 3). Por último, la Ley 8292 señala "Criterios mínimos que deberán observar la Contraloría General de la República y los entes u órganos sujetos a su fiscalización, en el establecimiento, funcionamiento, mantenimiento, perfeccionamiento y evaluación de sus sistemas de control interno" (Ley 8292, 2002, pág. 1).

Es imperativo definir el significado de riesgos que según la Directriz D-3-2005-CO-DFOE del SEVRI. Esta los señala como la "probabilidad de que ocurran eventos que tendría consecuencias sobre el cumplimiento de los objetivos fijados" (Contraloría General de la República, 2005, pág. 2); mientras que, según el Estándar Australiano, es "la posibilidad de que suceda algo que tendrá un impacto sobre los objetivos. Se lo mide en términos de consecuencias y probabilidades " (Australino, 1999, pág. 4). Según COSO, riesgo es "la posibilidad de que un evento ocurra y afecte en forma adversa el logro de los objetivos" (Ambrosone, 2007, pág. 4). Al identificar palabras clave de las diferentes definiciones, se evidencia el tema de impacto o afectación de los objetivos, que genera una influencia positiva o negativa en el plan estratégico de una organización.

A partir de lo anterior, se puede definir que la Administración de Riesgos se define como "la cultura, procesos y estructuras que están dirigidas hacia la administración efectiva de oportunidades potenciales y efectos adversos" (Australino, 1999, pág. 3). A su vez, y para la Contraloría General de la República, significa la cuarta actividad del proceso de valoración del riesgo. Esta última consiste en la identificación, evaluación, selección y ejecución de medidas para la administración de riesgos (en normativas técnicas, esta actividad también se denomina "tratamiento de riesgos") (Contraloría General de la República, 2005, pág. 1).

Otro concepto a definir es el modelo de gestión de riesgos financieros, que significa "la incorporación de sesgos sistemáticos u ocasionales en los criterios, supuestos, metodologías, bases de información o modelos de evaluación, que conducen a decisiones erróneas" (Bolaños, 2016, pág. 98). Un modelo se utiliza como ayuda para el pensamiento al organizar y clasificar conceptos confusos e inconsistentes, cuando se realiza un análisis de sistemas, se crea un modelo que muestra las entidades, las interrelaciones entre ellas. La adecuada construcción de un modelo ayuda a organizar, evaluar y examinar la validez de pensamientos. Con lo anterior, se define los riesgos financieros como "aquellos asociados a 
los productos y servicios que se pueden adquirir en los mercados financieros, de donde las empresas obtienen los recursos o los colocan" (Bolaños, 2016, pág. 97). Como ejemplo, lo define el Banco Ramón del Perú:

El modelo (...) es único, independiente y global y está integrado de forma proactiva en la gestión del negocio en todos los segmentos minoristas y mayoristas, lo cual permite ser una parte importante dentro de la relación con el cliente... (Bolaños, 2016, pág. 98).

Por lo tanto, el modelo de gestión de riesgos financieros brinda una opción que tendrá la administración financiera para mejorar los controles y establecer pautas, con el fin de cumplir con lo señalado en las disposiciones vigentes en materia de riesgos. Con lo anterior, puede definirse como una metodología de análisis cuantitativo y cualitativo de diferentes comportamientos de variables que pueden afectar un entorno sistémico tomando en consideración los riesgos que pueden afectar los productos y servicios en un mercado financiero. Existen diferentes tipos de modelos para la evaluación de riesgos; por ejemplo, los modelos estáticos (considerados solo en un momento en el tiempo de estudio) y los modelos dinámicos (toma en cuenta únicamente un periodo de tiempo de estudio) (Hernández, 2011, pág. 85).

Según Hernández, los modelos estáticos se basan normalmente en la contabilidad, tienen en cuenta magnitudes de referencia para el cómputo de los niveles de solvencia, variables como las cuentas del balance, las partidas integrantes de la cuenta de pérdidas y ganancias o la exposición a riesgos subyacentes de las inversiones. Una clasificación adicional de los sistemas estáticos los desglosa entre modelos simples y modelos de factores, en función del número de variables consideradas. Para ambos modelos existen pautas sobre las posiciones y factores que deberían incluirse en los cálculos para alcanzar un determinado nivel de confianza; en cambio, tienen la desventaja de reducir la valoración de la exposición al riesgo de la entidad a meras fórmulas y ratios.

Los modelos dinámicos se basan principalmente en proyecciones de flujos de caja y se pueden basar por medio de diferentes escenarios:

- Los modelos basados en escenarios implican una evaluación de la sensibilidad de la entidad al impacto de diversos escenarios en la valoración de sus activos, a través de la proyección de sus flujos de caja. La importancia de este método reside en la especificación de los escenarios y la forma de trasladar sus implicaciones. Principalmente, este test hace referencia al estudio de consecuencias con un amplio rango de parámetros que son variados simultáneamente, intentando analizar el impacto de determinados parámetros en función de la exposición específica de cada entidad. Los requerimientos de capitales mínimos se calcularían con base en el peor de los casos de los diferentes escenarios planteados.

- Sin embargo, en el caso de los modelos basados en principio no existen pautas sobre el sistema a emplear para cuantificar sus necesidades de capital, cuya valoración se basa en la utilización de modelos internos que siguen ciertos principios prefijados.

- Los modelos de base probabilista o stress testing, recogen el proceso por el que se evalúan un número definido de probabilidades estadísticas para determinar cuál es la peor combinación de parámetros y la pérdida que esa combinación produciría, intentando cubrir toda la gama de variables de riesgo reproducidas a través de distribuciones estadísticas y procedimientos de simulación. 
Según (Hernández, 2011) existen, además, otros modelos como el determinista y el probabilístico. El determinista "considera todos los procesos más importantes de un hecho y que las variables son conocidas con certeza" ( $p$. XX), en contra posición el modelo probabilístico, que es una

descripción matemática de un fenómeno aleatorio, es necesario concretar el espectro de resultados posibles. Un subconjunto del conjunto de todos los resultados posibles se denomina un evento. Si el grupo de resultados posibles es continuo, no puede asignarse generalmente una probabilidad positiva en cada resultado, pues la suma de las probabilidades no sería igual a 1. En tal caso, sin embargo, es posible asignar probabilidades a ciertos eventos. Si los resultados posibles son números reales, asignamos primero las probabilidades a los eventos con "resultado inferior a un número específico (p. 86).

El riesgo financiero "está relacionado con las pérdidas en el valor de un activo financiero, tales como un préstamo o una inversión" (Martínez, 2012, pág. 86). El autor señala dos tipologías de riesgos financieros: mercado y crédito. Por otra parte, se analiza como

la probabilidad de que ocurra un evento no deseado, en términos financieros se admite que el riesgo es una cualidad de las inversiones y de las operaciones financieras, en el tanto no existe certeza del cumplimiento de las condiciones pactadas en una inversión; así, cualquier elemento que atente contra el cumplimiento de las condiciones pactadas en un contrato financiero viene a ser un componente de riesgo. (Matarrita, 2013, p. 1).

Esta última señala de la existencia de tres tipos de riesgos: mercado, crédito y liquidez, que, según la definición anterior, se encuentra relacionada principalmente a entidades bancarias.
Al analizar cada uno de los riesgos mencionados anteriormente, se puede comprender que el riesgo de mercado viene a ser "el generado por instrumentos financieros o transacciones financieras provocado por fluctuaciones de precios, tasas de interés o tipos de cambio y aún las cotizaciones de acciones o de commoditys (productos básicos, como petróleo, soya, maíz, etc.)" (Matarrita, 2013, pág. 2). Lo anterior, siguiendo la misma conceptualización y tomando en cuenta las tasas de variación, resultan al afectar las tasas de interés sin alguna previsión y que da como resultado una desmejora en el precio de una determinada inversión para atender un determinado proyecto.

El riesgo proveniente del cambio en el precio de las acciones se manifiesta de dos formas: por una parte, está el riesgo de la caída en el precio de las acciones después de haber efectuado una compra y el riesgo de no poder vender todas las acciones que se desean liquidar. El riesgo de variación de precio de los commoditys (productos básicos, como petróleo, soya, maíz, etc.) se manifiesta cuando su comportamiento es distinto del esperado, tanto en la dirección como a la magnitud de los cambios.

El riesgo de crédito es el "riesgo generado por incumplimiento del cliente en transacciones financieras o de un emisor de instrumentos financieros; o bien, generado por el cambio en la credibilidad, es decir el aumento en la probabilidad de incumplimiento" (Matarrita, 2013, pág. 1). Este tipo de riesgo se clasifica de acuerdo c on las categorías de incumplimiento, ya sea de una cartera de clientes crediticios o de una cartera de activos financieros (por emisor). Cuando el deudor de un crédito no cumple, surge el riesgo de no poder recuperar los fondos que han sido prestados.

El riesgo de liquidez "... es aquel relacionado con la compraventa de un instrumento que se posee en que sea difícil su liquidación en el mercado y no se obtenga el monto esperado" (Matarrita, 
2013, pág. 4). Por su parte, el riesgo de liquidez o disponibilidad de fondeo (Availability Risk), es el riesgo de que la disponibilidad de recursos (fondeo) sea desventajosa o dificultosa para mantener un saldo de transacciones financieras. Tiene que ver con consideraciones de descalce en plazos.

Estos dos tipos de riesgos de liquidez están muy interrelacionados, pues la administración de la tesorería puede considerar realizar inversiones financieras. Estas inversiones pueden presentar dificultades de recuperación en caso de haberse programado con anticipación y en forma adecuada sus vencimientos.

El riesgo operacional (Matarrita, 2013, pág. 5) es generado por distintos factores de procedimientos de carácter operativo tales como:

- El error o fraude en el pago de fondo, la entrega de valores o la administración de las transacciones financieras.
- El defecto en los sistemas operativos.

- El error o fraude en la aplicación de los sistemas operativos.

Según la definición del Acuerdo de BIS, el riesgo operacional se define como el riesgo "de pérdida debido a la inadecuación o a fallas de los procesos, el personal y los sistemas internos o bien a causa de acontecimientos externos; esta definición incluye el riesgo legal, pero excluye el riesgo estratégico y el de reputación" (CITA).

La Ley 8292 de Control Interno de Costa Rica, establece la obligación y responsabilidad de la administración activa de disponer de un sistema de control interno (SCl) que busque la seguridad en materia de consecución de objetivos institucionales. Esa ley establece, en su artículo 14, que toda administración activa debe establecer un sistema de valoración de riesgos que identifique, analice, adopte y establezca mecanismos que minimicen los diferentes riesgos.

\section{FIGURA 1}

\section{ELEMENTOS DE GESTIÓN DE RIESGOS}

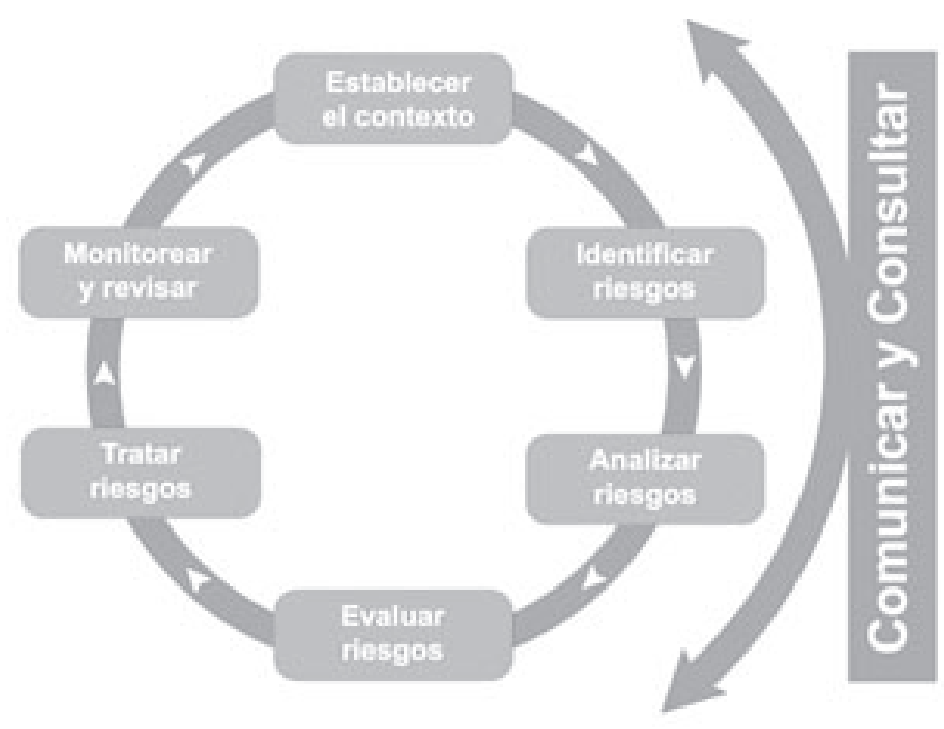

Fuente: Estándar Australiano (1999) 
TABLA 1

TABLA MATRIZ DE ANÁLISIS DE RIESGO CUALITATIVO

\begin{tabular}{|c|c|c|c|c|c|}
\hline \multirow{2}{*}{ Probabilidad } & \multicolumn{5}{|c|}{ Impacto } \\
\hline & Insignificantes 1 & Menores 2 & Moderadas 3 & Mayores 4 & Catastróficas 5 \\
\hline A (casi certeza) & H & $H$ & E & $E$ & $E$ \\
\hline B (probable) & M & $H$ & $H$ & $E$ & E \\
\hline ( (posible) & L & M & $\mathrm{H}$ & $E$ & $E$ \\
\hline D (improbable) & L & L & M & H & $E$ \\
\hline$E($ raro) & $L$ & $L$ & M & $H$ & $H$ \\
\hline
\end{tabular}

Fuente: Arce (2013), con base en estructura propuesta por el Curso de Gestión de Riesgos emitido por el CONARE para la Dirección Financiera de las Universidades Públicas.

Los Elementos de la Administración de riesgos son señalados en las Normas de Administración de Riesgos del Estándar Australiano (figura 1) (Australino, 1999).

El primer elemento es Establecer el contexto. En este se habla sobre la necesidad que tiene la organización de conocer o desarrollar el tema de la gestión estratégica (objetivos, metas, misión, visión, entre otros), conocer el organigrama y administración de riesgos en el cual tendrá lugar el resto de los procesos. Deberían establecerse criterios contra los cuales se evaluarán los riesgos y definirse la estructura del análisis.

Al Identificar riesgos, se debe conocer qué, por qué y cómo pueden surgir las situaciones como base para análisis posterior. El tercer elemento es Analizar riesgos, que consiste en determinar los controles existentes e identificar el rango de consecuencias potenciales y sus probabilidades de ocurrencia en el contexto de esos controles.

Como cuarto elemento, se tiene el Evaluar riesgos que compara niveles estimados de riesgos contra los criterios preestablecidos. Esto posibilita que los riesgos sean ordenados como para identificar las prioridades de administración. Si los niveles de riesgo establecidos son bajos, los riesgos podrían caer en una categoría aceptable y no se requeriría un tratamiento.
Para efectos de esta investigación y en concordancia con lo desarrollado en la universidad, por la unidad rectora en materia de control interno, se propone trabajar bajo el siguiente enfoque, presentado en la tabla 1.

La cantidad de categorías define la necesidad de atención en el estudio, el significado de las iniciales es:

- E. Riesgo extremo, requiere atención inmediata.

- H. Riesgo alto, necesita atención de la alta gerencia.

- M. Riesgo moderado, debe especificarse responsabilidad gerencia.

\section{- L. Riesgo bajo, administrar mediante procedi- mientos de rutina}

Un quinto elemento incorpora el Tratar riesgos. Acepta y monitorea los riesgos de baja prioridad. Para otros riesgos, este consiste en desarrollar e implementar un plan de administración específico que incluya consideraciones de fondeo. 
El sexto elemento es el Monitorear y revisar el desempeño del sistema de administración de riesgos y los cambios que podrían afectarlo. Finalmente, como séptimo elemento se encuentra Comunicar y consultar con interesados internos y externos según corresponda en cada etapa del proceso de administración de riesgos y concerniendo al proceso como un todo.

La gestión de administración de riesgos se puede aplicar en una organización según su área de atención y la necesidad de que se aplique según los niveles de la entidad. Se puede aplicar a nivel estratégico y operativo, así como a proyectos específicos, para contribuir con determinadas decisiones o áreas técnicas que requieran gestionar riesgos y posteriormente mitigarlos. En cada proceso es necesario obtener información con suficiente respaldo, que satisfaga a la auditoría interna o externa.

\section{METODOLOGÍA}

El enfoque utilizado en la investigación es cualitativo, se utilizó el método de casos para determinar la problemática por medio de un enfoque interpretativo de la información y a su vez crítico. El método de recopilación de datos se realizó por medio de entrevistas con las jefaturas que tiene a su cargo la Dirección Financiera; las preguntas utilizadas son de respuesta abierta, para tratar de obtener la mayor cantidad de información posible. Asímismo, se utilizó el método de observación por medio de un Curso Taller de Valoración de Riesgos realizado con la empresa Asesorías RRS en noviembre de 2011, donde se dispuso de treintaidós horas y que sirvió como insumo directo sobre el conocimiento y aplicación de la Ley 8292 de Control Interno y, además, tomar información para desarrollar más adelante el modelo a desarrollar.

El diseño de investigación de tipo no experimental se utiliza para establecer un análisis de casos con el fin de determinar los posibles riesgos que puede enfrentar la Dirección Financiera de la universidad. Con él, se pueden clasificar y establecer una metodología con resultados concretos en torno a la propuesta del modelo a proponer.

El tipo de diseño transaccional que se utilizó es generado por las características de recolección de datos en un único momento, a un grupo de personas que conocen de las actividades de la dirección. El diseño también es transaccional descriptivo, pues parte de la ubicación en una o diversas variables de riesgos que se tienen y con ellas permitir una serie de planes de acción para controlares y mejorar los riesgos asociados, con el fin de generar una propuesta para su aplicación.

La recolección de datos se realizó mediante la aplicación de un cuestionario con preguntas mixtas, con la opción de exponer respuestas cerradas o abiertas, para no dejar información importante en el proceso de recolección de datos. El instrumento busca obtener información básica que sirva como insumo al determinar información relevante de la dirección. Con ello, se busca establecer un parámetro del entorno, de cómo se encuentra y brindar criterios de riesgos para determinar una metodología inicial del marco metodológico del modelo de gestión de riesgos financieros.

La investigación se desarrolló con fuentes primarias: director financiero, como principal actor del área; jefes de las oficinas de presupuesto, tesorería, contabilidad y control de presupuesto, que figuran como segundos actores principales; y el criterio del vicerrector de turno. No se toma en cuenta al personal de la dirección debido a que era un tema que no se había trabajado a lo interno y que se da a conocer en una capacitación coordinada por CONARE en el mes de noviembre de 2011 por la empresa Asesorías RRS. De las fuentes secundarias, se analizó la Ley General de Control Interno No 8292 (2002), la Directriz General para el Establecimiento y Funcionamiento del Sistema Específico de Valoración de Riesgos Institucional 
(SEVRI, 2005) y el Estándar Australiano de Administración del Riesgo AS/NZS 4360:1999, así como estudios sobre aplicación y estructuración de riesgos, revistas e información alojada en la internet sobre el tema.

\section{DESARROLLO}

La Dirección Financiera está compuesta por cuatro oficinas: Presupuesto, Tesorería, Control de Presupuesto y Contabilidad. A partir de lo anterior, se aplican entrevistas con las jefaturas. Se toma en cuenta, además, la experiencia del Vicerrector Ejecutivo y Coordinador de la Oficina de Control Interno de turno, así como la información obtenida en el Taller de Valoración de Riesgos en el mes de noviembre de 2011. Con lo anterior se logra identificar ciertas debilidades, por ejemplo:

- Desde el punto de vista de la gestión estratégica, y a pesar de ser una Dirección que prácticamente tiene muy definido su rol dentro de la Universidad, no cuenta con una definición clara de su misión, visión y objetivos que, a su vez, se enmarque dentro del Plan de Desarrollo Institución y Políticas 2012-2015.

- No se encuentran desarrollados y aprobados por las instancias correspondientes los manuales operativos y sus respectivas funciones; además, carece de planes estratégicos y de acción para mejorar los procesos internos.

- No cuentan con un programa de Valoración del Riesgo Institucional que ayude a administrar los posibles riesgos existentes en dicha dirección.

Para cumplir con los objetivos planteados, se trató de plantear los elementos del riesgo desde el punto de vista práctico, en donde se generará cierta información básica cualitativa, con el fin de medir el nivel de riesgos de dicha dirección. Con lo indicado anteriormente, los resultados evidenciaron que existía un incumplimiento en lo indicado en la Ley 8292 y la Directriz del SEVRI de la Contraloría en lo correspondiente al artículo 14.

Al aplicar la directriz del SEVRI y establecer el contexto, se utiliza una matriz FODA (fortalezas, oportunidades, debilidades y amenazas), que es utilizado generalmente para la Planeación Estratégica, con el fin de desarrollar inicialmente un mapeo de riesgos de dicha dirección. Con su aplicación, se establece que la fortaleza más importante es el recurso humano, donde destacan como principales puntos la experiencia al realizar las funciones; la estabilidad que ofrece la institución para el funcionario; la formación del personal, esto debido a que la mayoría de funcionarios tienen grados académicos de bachillerato, licenciatura y maestría. Otras fortalezas ubicadas por oficina son:

- Oficina de Tesorería: controles en los procesos, el apoyo de la Dirección de Informática.

- Oficina de Control de Presupuesto: plazos de pago y renovación de equipo.

- Oficina de Contabilidad: estabilidad del personal.

- Vicerrectoría Ejecutiva: entrega e integridad del personal, estabilidad financiera, ya que se cumple con todas las responsabilidades de la Universidad.

- Programa de Valoración de la Gestión Administrativa y Riesgo Institucional (PROVAGARI, hoy PROCl): apoyo al personal por parte de la Administración General. 
Dentro de las debilidades, se identificaron los sistemas de información integrados. Esto debido a que la información desde que se presupuesta y hasta que se ejecuta el presupuesto en tres de las cuatro oficinas deben ingresarla manualmente en cada oficina, obligando a retardar y duplicar el proceso de registro, lo que motiva a desviar la atención del control y análisis de información. Una segunda debilidad la constituye la falta de creación, revisión y actualización de los manuales de procedimientos y procesos aprobados por la Dirección Superior. Según indica el Director Financiero, solo la oficina de Tesorería cuenta con los manuales, debido a que se desarrollaban por medio de una contratación externa que no concluyó el proceso en toda la dirección.

Otras debilidades identificadas son:

- Oficina de Tesorería: seguridad y falta de un fondo de cajero, para el manejo de efectivo.

- Oficina de Control de Presupuesto: conciliación a base de efectivo, por falta de tiempo, personal y herramienta para ejecutarla.

- Oficina de Contabilidad: competitividad salarial y desmotivación del personal.

- Vicerrectoría Ejecutiva: no hay rotación del personal, mayor capacitación al personal, procesos desactualizados. En la oficina de Tesorería hay exceso de confianza con el personal y, por ende, no se puede controlar el error humano. En Contabilidad se requiere mayor desarrollo del sistema de información para que se dediquen más al tema de análisis y generación de mejoras financieras. Control de presupuesto debe ejercer más fiscalización con el fin de evitar un pago doble. La oficina de Presupuesto debe apoyar más la parte financiera en cuanto a proyecciones y aportes en la generación de ingresos y trabajar más en conjunto con la oficina de Planificación con la formulación del Plan Operativo Anual.

- Dirección Financiera: poco crecimiento profesional en cuanto al control y manejo del presupuesto entre la Dirección Financiera y las Oficinas. Poco desarrollo profesional de las oficinas.

- $\quad$ PROVAGARI / PROCl: no existe una dependencia que se dedique a realizar estudios de impactos financieros y, en consecuencia, este se dedica a atender solicitudes de la Dirección Superior (Consejo Universitario, Rectoría y otros). No se trabaja el control del riesgo a nivel institucional; más bien, desde el punto de vista empírico y de forma reactiva, generalmente se reacciona ante una eventualidad y no se prevé desde antes.

Continuando con el análisis FODA, desde el punto de vista de oportunidades se obtuvo la que presenta el crédito que va a brindar el gobierno por parte del Banco Mundial a las Universidades Públicas. Lo anterior debido a que casi va a duplicar el presupuesto de la UNED, aspecto fundamental para demostrar capacidad de atención en proyectos mayores o iguales al presupuesto ordinario de la Universidad, lo que da como resultado un crecimiento en infraestructura, propuestas académicas y desarrollo o capacitación al personal de planta.

Dentro de las oportunidades que detallaron las oficinas están:

- Oficina de Tesorería: por las condiciones de atención de información, tiene la capacidad de conocer todo el acontecer de la Universidad. 
- Oficina de Control de Presupuesto: control de gastos y de ingresos permite brindar la información en el tiempo establecido por las diferentes unidades solicitantes, tanto externa como interna.

- Oficina de Contabilidad: la separación de la carrera universitaria puede dar un incentivo salarial adicional y el aprovechamiento de empleados calificados debido a su formación.

- Vicerrectoría Ejecutiva: potencial de crecimiento, por medio de la atracción de la nueva generación de la UNED, así como la comercialización de productos UNED para producción de nuevas fuentes de ingresos.

- Dirección Financiera: nuevas fuentes externas de financiamiento. Al parecer, hay alguna opción para la distribución del presupuesto del Fondo de Sistema y proyectos de ventas de servicios, como la venta de libros por internet, la editorial y el centro de idiomas.

- PROVAGARI / PROCl: aprovechar los servicios de educación a distancia, con el fin de capitalizar el conocimiento de los servicios en línea. La desconcentración de servicios hacia los Centros Universitarios donde se le brinda libertad presupuestaria a cada Centro, con el fin de que ejerzan el mismo según las necesidades presupuestarias. Mejoras de procesos y procedimientos según lo indicado en los informes de auditoría interna, principalmente con la atención de las recomendaciones técnicas.

Finalmente, en lo que corresponde a las amenazas, se puede afirmar que solo dos Oficinas tuvieron similitudes con respecto a la toma de decisiones que están fuera de la normativa financiera (Oficina de Tesorería y Control de Presupuesto).
Por lo tanto, se expone un resumen de lo recolectado por oficina:

- Oficina de Tesorería: la oficina jurídica no brinda el apoyo necesario para apoyar la tesorería y consecuencias negativas para la Universidad por hacer mal uso del presupuesto.

- Oficina de Contabilidad: fuga de personal capacitado por la Dirección, que el incentivo salarial al separar la carrera universitaria no sea oportuno. Que no se le saque el provecho a los empleados calificados.

- Vicerrectoría Ejecutiva: los ingresos propios sigan disminuyendo año con año.

- Dirección Financiera: el presupuesto UNED es muy limitado, desde que se elabora es deficitario, debido a que no tiene mucha maniobra para invertir en Activos Fijos, se considera que existe iliquidez, y a la vez se les ha aumentado el trabajo a ciertas oficinas tales como: control, tesorería y contabilidad.

- PROVAGARI/PROCl: la regulación o incumplimiento de normativas que vienen tanto del Gobierno Central (negociación del FEES), como de la Contraloría, pérdida de información o robo de información. Plazos de entrega de información al cliente externo de la Universidad.

La totalidad de los entrevistados mencionaron el conocimiento de la misión, aspecto que se comprobó con una serie de preguntas y una posterior revisión de este rubro. Sobre la visión, solo dos personas de las siete entrevistadas identificaron no conocerla. De los objetivos institucionales, solo dos personas no respondieron positivamente (una no lo conocía y otra no dio la entrevista en su momento). De las normativas 
TABLA 2

RIESGOS, CAUSAS Y ESCENARIOS NO SIMILARES, SEGÚN APLICACIÓN ENTREVISTA REALIZADA POR DEPENDENCIA, AÑ0 2013

\begin{tabular}{|c|c|c|c|}
\hline Dependencia & Evento o Riesgo & Causa & Escenario Posible \\
\hline $\begin{array}{l}\text { Oficina de } \\
\text { Tesorería }\end{array}$ & Seguridad. & Robo en Tesorería. & $\begin{array}{l}\text { Pérdidas humanas (integridad física y emocio- } \\
\text { nal), financieras. }\end{array}$ \\
\hline $\begin{array}{l}\text { Oficina de } \\
\text { Contabilidad }\end{array}$ & $\begin{array}{l}\text { Extravío de documen- } \\
\text { tación. }\end{array}$ & $\begin{array}{l}\text { En el proceso de trasiego y recibo de documentos, } \\
\text { se encuentran ubicadas varias dependencias y } \\
\text { personas, por lo que existe el riesgo de que se } \\
\text { traspapelen o se extravíen documentos. }\end{array}$ & $\begin{array}{l}\text { Errores en los registros contables e incumpli- } \\
\text { miento en el periodo de entrega de reportes. } \\
\text { Afectación a terceros. }\end{array}$ \\
\hline $\begin{array}{l}\text { Oficina de } \\
\text { Contabilidad }\end{array}$ & $\begin{array}{l}\text { Inadecuado control en } \\
\text { las bodegas generales de } \\
\text { libros. }\end{array}$ & $\begin{array}{l}\text { No existe un adecuado proceso de control de } \\
\text { existencias, debido a la falta de procedimientos en } \\
\text { el control y distribución de los libros a las depen- } \\
\text { dencias involucradas en el proceso de matrícula, } \\
\text { aunado a un mal manejo de bodega. }\end{array}$ & $\begin{array}{l}\text { Ocasiona errores en los registros contables y en } \\
\text { los reportes a otras dependencias. }\end{array}$ \\
\hline $\begin{array}{l}\text { Oficina de } \\
\text { Contabilidad }\end{array}$ & $\begin{array}{l}\text { Inadecuado control de } \\
\text { activos. }\end{array}$ & $\begin{array}{l}\text { No existe un adecuado manejo de información } \\
\text { con respecto a la custodia de los activos ya que } \\
\text { no se cumple lo estipulado en el reglamento para } \\
\text { dicho fin, esto en cuanto a las responsabilidades } \\
\text { de los funcionarios con respecto a los activos a su } \\
\text { nombre. }\end{array}$ & $\begin{array}{l}\text { Existe mucho rezago en el desempeño y rendi- } \\
\text { miento con respecto al mercado laboral actual. } \\
\text { Procesamiento y resultados tardíos. }\end{array}$ \\
\hline $\begin{array}{l}\text { Oficina de } \\
\text { Contabilidad }\end{array}$ & $\begin{array}{l}\text { Carencia de equipo } \\
\text { adecuado para las labores } \\
\text { cotidianas de la depen- } \\
\text { dencia. }\end{array}$ & $\begin{array}{c}\text { No se cuenta con equipo actualizado para las } \\
\text { labores diarias de la dependencia. }\end{array}$ & \\
\hline \multirow{4}{*}{$\begin{array}{l}\text { Vicerrector } \\
\text { Ejecutivo }\end{array}$} & Malversación de Fondos. & Control. & Pérdida de dinero. \\
\hline & Desmotivación. & Monotonía en el trabajo. & Pérdida del recurso humano. \\
\hline & Inversión riesgosa. & Invertir en puestos no autorizados. & Pérdida de dinero. \\
\hline & Falta análisis. & Falta de tiempo. & Toma de decisiones. \\
\hline $\begin{array}{l}\text { Dirección } \\
\text { Financiera }\end{array}$ & $\begin{array}{l}\text { Presupuesto Ordinario sin } \\
\text { aprobar. }\end{array}$ & $\begin{array}{l}\text { No existe presupuesto ordinario en el periodo de } \\
\text { ejecución. Problema de ejecución presupuestaria, } \\
\text { puede darse por negligencia, problemas de los } \\
\text { sistemas de información. }\end{array}$ & $\begin{array}{l}\text { Se vea obligada a utilizar el presupuesto del } \\
\text { año anterior, esto debido a que no se presentó } \\
\text { en tiempo el presupuesto ordinario. }\end{array}$ \\
\hline \multirow{2}{*}{ PROVAGARI } & Presupuesto. & Mala formulación presupuestaria. & Problemas en la ejecución presupuestaria. \\
\hline & Inversiones. & Malas decisiones. & Pérdidas a nivel cambiario. \\
\hline
\end{tabular}

Fuente: Arce (2013). Entrevistas realizadas a los jefes, director de la Dirección Financiera y Vicerrector Ejecutivo.

(estatutos orgánicos, creación de la UNED, normativas y procedimientos, estatuto de personal, reglamentos del área administrativa y de recursos humanos), solo una persona indicó que aplica todas las señaladas; las demás respondieron que si la aplicaban.
Del grado de aprobación para la aplicación de procesos y procedimientos, solo dos oficinas cuentan con la aprobación del 100\% de los procesos (control de presupuesto y tesorería). Las demás se encuentran en pleno desarrollo. 
En la etapa de identificación de riesgos, se lograron establecer preliminarmente algunos eventos o riesgos que afectan a la Dirección Financiera y que pueden generar riesgos de incumplimiento de los objetivos de cada oficina.

Dentro de los eventos similares que se identificaron, en la Dirección se encontró como número uno el "error humano". Este puede ser generado por las siguientes causas: errores en pago a proveedores, errores en montos reportados, omisión de pasos (falta de firmas, sellos, V.B.), errores involuntarios al digitar, información falsa, falta de tolerancia por parte del funcionario, en errores de cálculo en la formulación del presupuesto, malas anotaciones contables, problemas de revisión de información, incumplimiento del procedimiento de tesorería, mala digitación en las transferencias y malas decisiones en inversiones (divisas).

Dentro de las posibles consecuencias que podrían enfrentar son las demandas por parte de proveedores y personas en particular por incumplimiento de pagos o acciones que no se tomen en el plazo establecido. Ocasionan atrasos por incumplimiento de las fechas de registro y en reportes generados a otras oficinas, errores en los registros contables e incumplimiento en el periodo de entrega de reportes y, con ello, afectación a terceros, pérdida de dinero, malas decisiones en cualquier nivel de la ejecución, información no contable, falta de tiempo, se deben realizar correcciones inmediatas a los presupuestos ordinarios o extraordinarios, mala información en los informes de los Estados Financieros.

El segundo riesgo similar es de "Sistemas de Información" y, dentro de las posibles causas, los retrasos en el proceso operativos de la Universidad. Si ingresa un hacker a los sistemas, puede adquirir información privada, por omisión en el mantenimiento de los procesos informáticos $u$ errores en la definición de los parámetros relacionados con el cálculo de la depreciación de activos, traslado de información entre relaciones bancarias entre otras, carencia de un sistema de información integrado, con lo cual hace que la información sea digitalizada hasta tres veces y con ello exista pérdida de tiempo en aspectos operativos del proceso administrativo y dejar de lado el trabajo analítico que tanto requiere la Universidad.

Con respecto a las consecuencias, la información financiera podría ser difícil de conciliar desde el punto de vista contable-presupuestario, la entrada de virus, mal uso del sistema y no brindar la información en tiempo, por error o por factores humanos. Asímismo, se dan errores en los registros contables, en reportes a otras dependencias, pérdidas de horas "hombre" y tendencia a dedicar el tiempo en aspectos operativos y no analíticos de la información que resulta al finalizar cada periodo financiero. Otra consecuencia es la confiabilidad en los sistemas de información, al no ser sistemas integrados generan, como el riesgo de error humano, el incremento de errores en registros y además la eficiencia de los sistemas de información. A lo externo de la Universidad generan problemas con entes externos, por emitir tarde la información y no contar con un sistema eficiencia y eficaz para la generación de información lo que ocasiona posibles demandas legales, causas por multas, disminución del presupuesto, entre otras.

\section{CONCLUSIONES}

Luego de los resultados obtenidos y tomando en consideración lo recolectado con base en los dos primeros objetivos, se obtuvo información relevante al aplicar la recolección y análisis de algunos riesgos identificados. Esta sirvió de prueba para sustentar la validación inicial de la propuesta a implementar. Se debe considerar que el modelo inicialmente es estático, pero puede llegar a desarrollarse en uno dinámico dependiendo del grado de maduración y su realimentación a futuro; por ejemplo, el desarrollo de un modelo 
de riesgo de crédito o de inversión cuantitativo, que logre implementar el Valor en Riesgo de una inversión y que ayude en la toma de decisiones en la Oficina de Tesorería. La validación indicada permitió obtener información relevante para la propuesta, con las siguientes acciones:

- Una directriz sobre la aplicación del sistema de valoración de riesgos a lo interno de la DF.

- Un marco estratégico de la DF, incorporando la administración de riesgos.

- El responsable de la gestión del riesgo en la DF, según lo dispuesto en la Ley 8292 General de Control Interno, en el capítulo III donde se menciona el tema de la Administración Activa en el artículo 12 de Deberes del jerarca y de los titulares subordinados en el sistema de control interno.

- La metodología para su respectiva aplicación de las etapas de la Gestión del Riesgo, según lo propuesto en la Ley 8292 y la Directriz del SEVRI emitida por la Contraloría General de la República.

- Acciones concretas para capacitar e interiorizar el personal interno de la Dirección.

Para desarrollar el Modelo de Gestión de Riesgos en la DF de la UNED, se debe iniciar con la solicitud formal de asesoría al área responsable de Control Interno Institucional, dependencia que tiene definida dentro de sus funciones el apoyar el desarrollo del sistema de control interno en la UNED. Dicha entidad será la encargada, en conjunto con la DF, de abordar el proceso de desarrollo e implementación del Marco Metodológico (ver Figura 2).
Consta de tres etapas dependientes una de la otra y una cuarta etapa que incluye la revisión del diseño. Todas ellas tienen como objetivo controlar o mitigar aquellas actividades riesgosas tanto internas como externas que comprometan el accionar operativo y financiero de la DF y de la Universidad.

En la etapa de Organización Interna se define o crea un equipo de trabajo (comisión interna) que será el encargado o experto de la gestión del riesgo en la dirección. Se define un coordinador general; puede excluirse al Director Financiero, aunque es importante que conforme el equipo de trabajo. Los integrantes deben realizar un proceso de formación por medio de talleres, formación profesional o asesoría dirigida por un especialista en el tema; se recomienda utilizar la metodología constructivista "aprender-haciendo", para obtener resultados inmediatos en el desarrollo de la gestión del riesgo institucional. Se recomienda trabajar, al menos, los siguientes temas:

- Aprendizaje y aplicación del tema sobre riesgos institucionales, su definición y funcionamiento, aplicación de elementos.

- Analizar las leyes y reglamentos emitidos por la CGR.

- Analizar la gestión estratégica de la Universidad.

En la etapa de Planificación del Entorno se trabajará por componentes estratégicos para alcanzar el objetivo, el cual requiere, principalmente, entender el contexto en donde se aplica la gestión de riesgos. En esta etapa se debe realizar una revisión documental completa de la DF, donde se recolecta información relacionada con los objetivos de la Institución, acuerdos del Consejo Universitario que afecten directa o 


\section{FIGURA 2 \\ MARCO TÉCNICO PARA EL MODELO DE GESTIÓN DE RIESGOS PARA DF}

Fuente: Arce (2013)

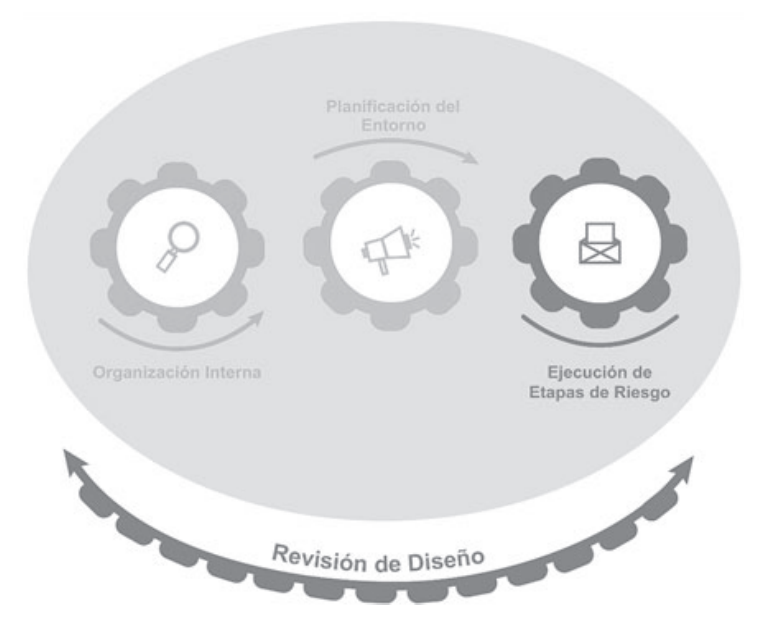

indirectamente a la DF, directrices de Rectoría, procedimientos de la DF y sus oficinas adscritas, entre otras que pueda considerar el equipo organizacional. Adicionalmente, se debe tomar en cuenta el entorno externo (leyes, reglamentos, coyuntura económica, solicitudes de la Contraloría General de la República, solicitudes del gobierno de la república, contratos establecidos entre UNED y sector público-privado, ya sea Bancos del Estado, instituciones descentralizadas, semiautónomas y empresas privadas que brinden servicio a la UNED, entre otras identificadas por el equipo de trabajo).

En esta etapa es importante desarrollar un análisis de gestión estratégica empresarial, por medio de la técnica de FODA, con el fin de establecer posteriormente las áreas o procesos que se encuentran con mayor riesgo (debilidades y amenazas). Así, también es necesario tomar en cuenta aquellos procedimientos internos que ayuden a profundizar más y a obtener mayor información sobre la DF. Se recomienda coordinar, en caso necesario, con un experto en planificación estratégica del

Centro de Planificación y Evaluación Institucional de la Vicerrectoría de Planificación, para que capacite a los colaboradores sobre el uso, aplicación y desarrollo de este tipo de instrumento.

Finalmente, es esencial determinar el apetito al riesgo o cantidad de riesgo aceptable según parámetros establecidos a lo interno de la Universidad, que por lo general utilizan categorías de tres a cinco resultados cualitativos según la decisión a lo interno de la UNED. Estos pueden ser riesgos altos, medios o bajos e, inclusive, intermedios, lo cual abarca el riesgo medio-alto y medio-bajo.

La etapa Ejecución de etapas del Riesgo se genera a partir de identificar, analizar o evaluar. También implica la alineación y actividades de control y comunicación de riesgos. Antes de aplicar cada sub-etapa, se debe analizar la estructura de riesgos (nombre del riesgo, factor y descripción) definida a nivel institucional por la oficina de Control Interno. 
Es necesario retomar el análisis FODA y los procedimientos, para generar una lista de eventos (ique pueden ocurrir en la DF?) que permita hacer valoraciones y establecer prioridades de atención al riesgo (determinar el riesgo más importante y generar la primera alerta de atención). Esto es insumo para la primera sub-etapa.

La primera sub-etapa parte al identificar el riesgo, donde se indagarán aquellas acciones internas o externas que pueden afectar cualquier actividad a lo interno de la DF. En este se plantea la siguiente pregunta: ¿Qué puede suceder para obstaculizar el desarrollo de las actividades de la Dirección Financiera? ¿Cómo puede verse afectada? Con las dos preguntas anteriores se ubica el riesgo y posibles factores. Para determinar las consecuencias se trabaja con las siguientes preguntas: ¿Cuáles son los efectos o impactos posibles al identificar el riesgo?

Luego, se clasifica el riesgo según la estructura de riesgos institucional; por ejemplo, pueden ser riesgos de entorno, riesgos estratégicos o de supervisión del esquema de negocio, riesgos de negocio u operacionales y riesgos de asignación de recursos.

La siguiente sub-etapa de análisis o evaluación del riesgo permite determinar la probabilidad y el impacto/consecuencia del riesgo que genera el nivel de cada riesgo identificado. Existen instituciones que trabajan con matrices de tres por tres o de cinco por cinco, esto depende del nivel de resultados que desean obtener. Para esta ocasión, se recomienda trabajar con una matriz de tres por tres, donde la probabilidad (posibilidad de que ocurra un evento o riesgo identificado) se mida con las siguientes categorías: probable, posible e improbable; el impacto/consecuencia (efecto directo que puede ocasionar la materialización del riesgo) se mide en insignificante, moderada y catastrófica. Ambas categorías pueden ser descritas cualitativamente y cuantitativamente; asimismo, permiten acotar el grado de objetividad que se pueda adquirir al recolectar los resultados. Se debe tener claro el apetito al riesgo que tiene la Universidad con respecto al riesgo, con el fin de obtener o generar valor a todo el sistema UNED y definir sus acciones de mejora o atención para controlar o mitigar el riesgo institucional.

En la sub-etapa de alineación y actividades de control trae consigo elementos para priorizar la atención de los riesgos según lo establezca la Dirección Financiera. La alineación tiene que ver con la aceptabilidad o no del riesgo y se trabajan las definidas por la Oficina de Control Interno Institucional. En este caso, pueden ser aceptables, de reducción, de evitarlos o de compartir los riesgos. Estas acciones serán definidas con anterioridad por dicha oficina y resultan del mismo apetito al riesgo. Ahora bien, en caso de que se acepten, estas no traen consigo un seguimiento continuo; al evitarlos o reducirlos, deben generarse planes de acción incluido el responsable, fechas e indicadores de atención y de seguimiento exclusivo por la DF y, en caso de compartir el riesgo, adicionalmente a los planes de acción definidos, estos deben ser coordinados y trabajados entre las dependencias involucradas.

En las actividades de control es importante tener claro lo siguiente:

- ¿Cómo se recopilan los controles de los riesgos identificados?

- ¿Qué se entiende por documentar un control?

- ¿Qué se entiende por aplicar un control?

- ¿Qué se entiende por efectividad del control?

Al evaluar los controles, se enlistan los controles existentes y cuáles, en teoría, debería tener cada riesgo evaluado. Los controles pueden ser 
evaluados por medio de factores cualitativos. Uno muy sencillo de utilizar es, por ejemplo, el Sí o No. Sin embargo, se debe tomar en cuenta que al elegir este último, debe desarrollarse un plan de mejora que mitigue o controle el riesgo.

La sub-etapa final de comunicación de riesgos es una actividad que trata, principalmente, de trasladar los planes de acción a los involucrados en los procesos analizados, con el fin de establecer responsables y emitir una comunicación eficaz en todo sentido en la DF. En este plan de tratamiento, se define como mínimo los responsables, resultado esperado (mejoras esperadas), estado de cumplimiento de las acciones de mejora y cronograma de atención de los eventos.

Esta sub-etapa parece ser muy sencilla. En realidad, la atención eficiente permite compartir y concientizar los planes de acciones desarrollados en conjunto con el equipo de trabajo. Lo anterior se hace para su debida atención en tiempo y plazo a lo interno de la dirección y que resulte significativo para el cumplimiento de lo planteado en la etapa de Organización.

En la etapa de Revisión del Diseño se da el seguimiento y realimentación del proceso de gestión de riesgos. El proceso involucra la eficiencia y eficacia de la aplicación del modelo. Para ello, es importante el análisis de resultados obtenidos, el grado de madurez que ha adquirido en su implementación en la DF y que será cíclico para mejorar los procesos. En casos necesarios, es importante plantear acciones concretas para:

- Brindar seguimiento a los participantes del equipo de expertos, con el fin de mantener a todos actualizados.

- Establecer planes de capacitación o talleres para los colaboradores de toda el área Financiera, con el fin de apropiar del sistema a los integrantes de la Dirección y compartir experiencias del modelo aplicado.

- Generar periodos de realimentación del proceso de gestión de riesgos, con el fin de mejorar y establecer medidas correctivas, conforme se tome mayor experiencia en administración del riesgo integral en la Universidad, se adquirirá mayor conciencia en el seguimiento del riesgo y se desarrollarán metodologías más específicas, según requerimientos de las Oficinas y la Dirección Financiera.

Será cíclica durante todo el proceso de gestión de riesgo, ya que es importante actualizar todo el proceso, además de recopilar información que sirva para la toma de decisiones y, finalmente, para generar valor y cumplir con los objetivos institucionales de la UNED.

\section{BIBLIOGRAFÍA}

Acuerdo SUGEF 2-10, Artículo 9, del acta de la sesión 862-2010. Celebrada el 25 de junio del 2010. Publicado en el diario oficial La Gaceta N ${ }^{\circ} 137$ del 15 de julio del 2010. Superintendencia General de Entidades Financieras.

Ambrosone, M. (2007). La administración del riesgo empresarial: una responsabilidad de todos-el enfoque coso.

Bravo Mendoza, O., \& Sánchez Celis, M. (2006). Gestión Integral de Riesgos. Tomo I. Bogotá, Colombia: Bravo \& Sánchez.

Caja Costarricense del Seguro Social. (CCSS). (2007). Guía para la aplicación del Sistema Específico de Valoración de Riesgo (SEVRI) en los procesos. Costa Rica: CCSS.

Caja Costarricense del Seguro Social. (CCSS). (2007). Modelo de Estructura de Riesgos Institucionales. Costa Rica: CCSS.

Contraloría General de la República. (2005). Directrices Generales para el Establecimiento y Funcionamientos del Sistema Específico de Valoración 
del Riesgo Institucional (SEVRI) D-3-2005-CODFOE.

Cuello, R., Pallares, L., \& Wehdeking, E. (2008). Aplicación del Estándar Australiano de Administración del Riesgo AS/NZS 4360:1999 en la empresa GEGELCA. Pensamiento y Gestión, (25), 94-112.

Hernández, R. (2011). Metodología financiera de gestión y cuantificación de riesgos de las entidades aseguradoras. Pecvnia: Revista de la Facultad de Ciencias Económicas y Empresariales, Universidad de León, (2011), 81-107.

Hernández, R., Fernández, C., \& Baptista, P. (2013). Metodología de la Investigación. México: McGraw Hill.

Ley General de Control Interno N ${ }^{\circ} 8292$ para el Sector Público y el Privado, 2002.

Lizarzaburu, E. R., Berggrun, L., \& Quispe, J. (2012). Gestión de riesgos financieros. Experiencia en un banco latinoamericano1. Estudios Gerenciales, 28(125), 96-103.

Martínez, I. (2012). Definición y Cuantificación de los Riesgos Financieros. Revista Actuarios, (30), 26-29.

Matarrita, R. (2013). Teoría del Riesgo Financiero. Costa Rica: FUNDEVAL. Recuperado de https:// es.scribd.com/document/267724614/Teoria-Del-Riesgo

Ojeda, E. (2007). Estándar Australiano de Administración del Riesgo AS/NZS 4360:1999.

Universidad Nacional de Colombia. (2013). Dirección General de Servicios Académicos. Recuperado de http://www.virtual.unal.edu.co

Recibido: 10 de agosto de 2018

Aceptado: 04 de abril de 2019 